\title{
PARTO NORMAL E INTERVENÇÕES OCORRIDAS EM UMA MATERNIDADE PÚBLICA
}

\author{
NORMAL CHILDBIRTH AND INTERVENTIONS \\ IN A PUBLIC MATERNITY
}

\section{PARTO NORMAL E INTERVENCIONES EN UNA MATERNIDAD PÚBLICA}

\author{
Luana Rocha Pereira ${ }^{1}$ \\ Gilcynara Maria Moura Rodrigues ${ }^{2}$ \\ Elisângela da Silva Ferreira ${ }^{3}$ \\ Ingrid Nicolle Monteiro Barros ${ }^{1}$ \\ Marcia Simão Carneiro ${ }^{4}$ \\ Lorena Saavedra Siqueira
}

Como citar este artigo: Pereira LR, Rodrigues GMM, Ferreira ES, Barros INM, Carneiro MS, Siqueira LS. Parto normal e intervenções ocorridas em uma maternidade pública. Rev baiana enferm. 2019;33:e-32631.

Objetivo: analisar a relação entre as intervenções realizadas durante o trabalho de parto e a duração da fase ativa em parturientes internadas. Método: estudo documental, tipo levantamento de dados, com abordagem quantitativa, realizado em uma maternidade no Pará, Brasil. A análise dos dados ocorreu por meio de estatística descritiva. Resultados: identificadas as intervenções: amniotomia (6,1\%), ocitocina (64,2\%) e episiotomia (16,7\%). A relação das intervenções com a duração da fase ativa do trabalho de parto ocorreu com maior frequência em mulheres com menos de 5 horas na fase ativa. Conclusão: as intervenções realizadas em partos de risco habitual e no período de fase ativa menor que 5 horas não encontraram sustentação teórica e remeteram ao modelo biomédico.

Descritores: Enfermagem Obstétrica. Parto Normal. Trabalho de Parto. Obstetrícia.

Objective: to analyze the relation between the interventions performed during labor and the duration of the active phase in hospitalized parturients. Method: a documentary study, of the data collection type, with a quantitative approach, performed in a maternity hospital in Pará, Brazil. Data analysis was performed using descriptive statistics. Results: the following interventions were identified: amniotomy (6.1\%), oxytocin (64.2\%) and episiotomy (16.7\%). The relation between the interventions and the duration of the active phase of labor occurred most often in women with less than 5 bours in the active phase. Conclusion: the interventions performed in habitual risk deliveries and in the active phase period with less than 5 hours did not find any theoretical support and referred to the biomedical model.

Descriptors: Obstetric Nursing. Normal Childbirth. Labor. Obstetrics.

Objetivo: analizar la relación entre las intervenciones realizadas durante el trabajo de parto y la duración de la fase activa en parturientas internadas. Método: estudio documental, del tipo recolección de datos y con enfoque cuantitativo, realizado en una maternidad de Pará, Brasil. Los datos se analizaron por medio de estadística

\footnotetext{
Enfermeira. Pesquisadora independente. Belém, Pará, Brasil.

Estudante de Enfermagem. Universidade Federal do Pará. Belém, Pará, Brasil.mouranaah05@gmail.com

Enfermeira. Mestre em Biologia dos Agentes Infecciosos e Parasitários. Professora da Universidade Federal do Pará. Belém, Pará, Brasil. licalipe8@yahoo.com.br. https://orcid.org/0000-0002-7983-7799

4 Enfermeira. Doutora em Educação. Professora da Universidade Federal do Pará. Belém, Pará, Brasil.

Enfermeira. Especialista em Residência em Enfermagem Obstétrica. Belém, Pará, Brasil. https://orcid.org/0000-0003-0870-0522
} 
descriptiva. Resultados: se identificaron las siguientes intervenciones: amniotomía (6,1\%), oxitocina (64,2\%) y episiotomia (16, 7\%). La relación entre las intervenciones y la duración de la fase activa del trabajo de parto se hizo presente con mayor frecuencia en mujeres con menos de 5 boras en la fase activa. Conclusión: las intervenciones realizadas en partos de riesgo habitual y durante períodos de la fase activa de menos de 5 horas no encontraron sustento teórico alguno y se remitieron al modelo biomédico.

Descriptores: Enfermería Obstétrica. Parto Normal. Trabajo de Parto. Obstetricia.

\section{Introdução}

O cuidado à mulher durante o trabalho de parto e parto, além de pressupor interação, deve adotar técnicas sustentadas por evidências científicas que contribuam para a autonomia e o empoderamento da mulher no cenário do parto e nascimento, assim como para a diminuição da morbidade e mortalidade materna e perinatal.

O modelo obstétrico brasileiro passa por um período de transição entre o modelo biomédico e o humanizado. De acordo com o modelo biomédico, o trabalho de parto e parto é visto como evento de risco para o binômio mãe-feto. Por isso, necessita do ambiente hospitalar com tecnologia para possíveis intervenções ${ }^{(1)}$.

Quanto à realização dos partos em ambientes hospitalares, conforme as Diretrizes Nacionais de Assistência ao Parto Normal ${ }^{(2)}$, esse ambiente é propício para que a mulher sofra intervenções, de forma indiscriminada, a exemplo da episiotomia, do uso de ocitocina, da realização de amniotomia e indicação de cesárias desnecessárias. As Diretrizes evidenciam que, durante o processo parturitivo, o excesso de intervenções reforça a visão biomédica do parto e desconsidera os aspectos emocional, cultural e humano. Desse modo, o cuidado profissional durante o parto e o nascimento deve considerar as singularidades de cada mulher e família para além do processo de parir e nascer ${ }^{(2)}$.

É válido ressaltar que o modelo de atenção ao parto com uso excessivo de intervenções, como a amniotomia, o uso de ocitocina no trabalho de parto e a episiotomia, não encontra respaldo em estudos internacionais ${ }^{(3)}$.

A ocitocina é um hormônio com ação central no trabalho de parto, por ser responsável pelo estímulo das contrações uterinas. Por isso, seu uso está associado a uma série de outras intervenções, nomeadas como "efeito cascata", atualmente questionado e colocado em pauta nos debates sobre os direitos sexuais e reprodutivos, assim como nas discussões sobre a autonomia da mulher sobre seu corpo, também no momento do parto ${ }^{(4)}$.

Quanto à realização da amniotomia, a Organização Mundial da Saúde (OMS) ${ }^{(5)}$ define que é o rompimento precoce das membranas amnióticas e esclarece que, embora possa encurtar a primeira fase do trabalho de parto, não está demonstrada diferença significativa em outros resultados clínicos importantes.

No que se refere à episiotomia, trata-se de procedimento cirúrgico que objetiva aumentar a abertura vaginal por uma incisão no períneo durante o período expulsivo ${ }^{(6)}$. Nos últimos anos, estudos publicados demonstraram que tanto as indicações de episiotomia quanto as técnicas variam conforme o contexto e a equipe profissional ${ }^{(7)}$.

Assim, observa-se que há necessidade que os profissionais de saúde utilizem práticas obstétricas humanizadas, pois, em muitos casos, a maioria das intervenções são desnecessárias e acabam trazendo malefícios às parturientes de risco habitual $^{(8)}$.

Nessa perspectiva, este estudo tem como objetivo analisar a relação entre as intervenções realizadas durante o trabalho de parto e a duração da fase ativa em parturientes internadas.

\section{Método}

Trata-se de um estudo documental, retrospectivo, do tipo levantamento de dados, com abordagem quantitativa, realizado em maternidade 
no estado do Pará, Brasil. A amostragem foi probabilística, sendo o tamanho da amostra obtido pela fórmula para cálculo de proporções em populações finitas, com base na média de partos normais de risco habitual ocorridos entre os anos de 2015 e 2016.

Foram incluídos 358 prontuários com base nos critérios de inclusão: prontuários de parturientes no período puerperal e que passaram por parto de risco habitual. Foram critérios de exclusão: prontuários de parturientes fora do período puerperal e que tiveram parto de alto risco. Os dados coletados foram analisados com o recurso da estatística descritiva, por meio de número absoluto e relativo. O estudo foi aprovado pelos Comitês de Ética e Pesquisa (CEP), CAAE 72565317.0.0000.0018 e 72565317.0.3001.5171.

\section{Resultados}

A relação entre as intervenções realizadas durante o trabalho de parto e a duração da fase ativa em parturientes demonstra que, nos casos de rotura das membranas, cerca de 93,3\% ocorreu de forma espontânea. O uso de ocitocina durante o trabalho de parto teve predominância de 64,2\% e a realização de episiotomia ocorreu em 13,7\% dos casos. A assistência sem quaisquer dessas intervenções durante o trabalho de parto ocorreu em 28,2\% dos prontuários estudados. Esses dados estão informados na Tabela 1.

Tabela 1 - Descrição das intervenções realizadas durante o trabalho de parto em parturientes internadas em uma maternidade de Belém. Belém, Pará, Brasil - 2015-2016

\begin{tabular}{l|l|c|c}
\hline Variável & \multicolumn{1}{|c|}{ Descrição } & $\mathbf{n}$ & $\begin{array}{c}\text { Frequência } \\
\text { (\%) }\end{array}$ \\
\hline Rotura das membranas & Espontânea & 334 & 93,3 \\
& Induzida & 22 & 6,1 \\
& Sem Informação & 2 & 0,5 \\
Ocitocina & Sim & 230 & 64,2 \\
& Não & 125 & 34,9 \\
& Sem informação & 3 & 0,8 \\
Episiotomia & & & 16,7 \\
& Sim & 60 & 83,2 \\
Total & Não & 298 & 100 \\
\hline
\end{tabular}

Fonte: Elaboração própria.

Para analisar a relação entre as intervenções realizadas durante o trabalho de parto e a duração da fase ativa nas parturientes, delimitou-se o tempo de duas formas: menor ou igual a 5 horas e maior que 5 horas. Entre as parturientes que estavam em trabalho de parto ativo por um tempo menor ou igual a 5 horas, 6,9\% foram submetidas à amniotomia; 44,6\%, ao uso de ocitocina; e 11,1\%, à episiotomia. Quanto às parturientes que permaneceram na fase ativa por mais de 5 horas, 1,9\% foram submetidas à realização de amniotomia; 18,9\%, à ocitocina; e 5,5\%, à episiotomia. O Gráfico 1 permite a visualização desses resultados. 
Gráfico 1 - Relação da fase ativa do trabalho de parto com as intervenções

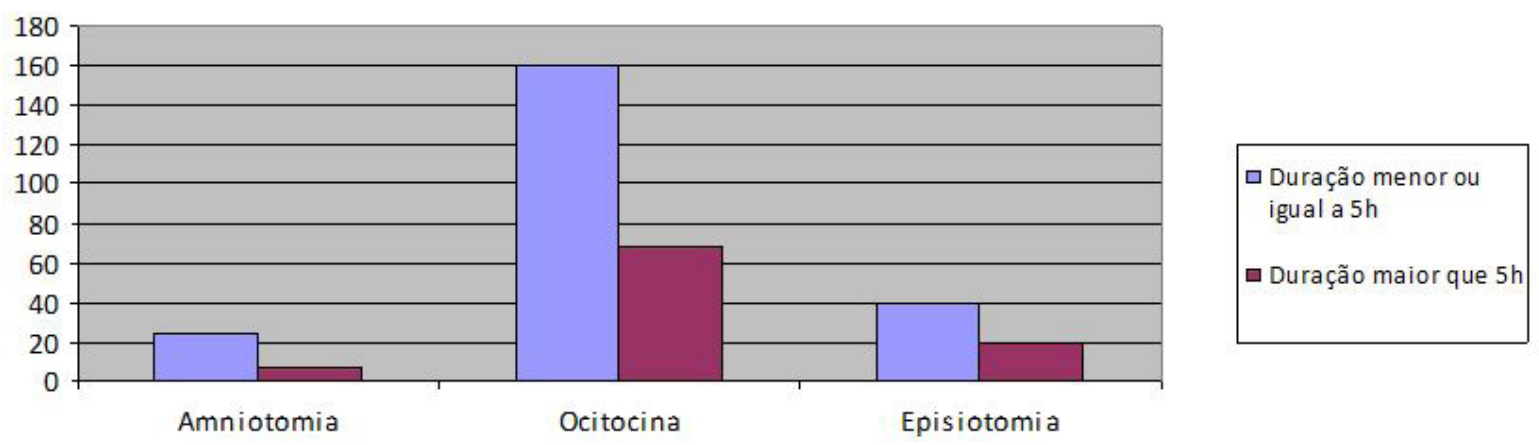

Fonte: Elaboração própria.

\section{Discussão}

A necessidade de acelerar o trabalho de parto e parto, muitas vezes, é demanda do profissional de saúde, à medida que o controle do tempo e a imposição do processo explicam o índice excessivo de intervenções. No hospital, esse processo tem solução de continuidade, por meio de intervenções que não se baseiam em evidências científicas, e submetem as mulheres brasileiras, de todos os grupos socioeconômicos de risco habitual, à exposição desnecessária e aos riscos de iatrogenia no parto ${ }^{(3)}$.

Segundo as recomendações da Organização Mundial de Saúde (OMS), o trabalho de parto não pode ser acelerado até que atinja uma dilatação cervical de cinco centímetros. Essas recomendações visam prevenir os efeitos adversos maternos e perinatais iatrogênicos, minimizar intervenções desnecessárias e melhorar a experiência do parto materno ${ }^{(5)}$. Ressalta-se que, nos prontuários analisados, as parturientes estavam com dilatação uterina maior ou igual a quatro centímetros.

No que tange às intervenções, sobre a amniotomia, as evidências demonstraram que essa intervenção é pouco realizada, conforme as orientações da Organização Mundial de Saúde, que recomenda a não realização desse procedimento de forma rotineira, com vistas à prevenção de atraso no trabalho de parto ${ }^{(5)}$. No entanto, os resultados sobre a realização de amniotomia podem ter influência no número de parturientes admitidas em trabalho de parto com bolsa amniótica rota, critério que não fez parte da exclusão do estudo.

Com relação à infusão de ocitocina para conduzir ou acelerar o trabalho de parto, é um dos procedimentos mais realizados na prática obstétrica. Essa intervenção é frequentemente utilizada para aumentar a atividade uterina em casos que ocorrem falhas na evolução do trabalho de parto, para viabilizar a progressão do parto vaginal ${ }^{(9)}$.

Neste estudo, evidenciou-se um percentual elevado do uso de ocitocina em parturientes durante o trabalho de parto. Sobre o uso dessa medicação sintética, a $\mathrm{OMS}^{(10)}$ não recomenda para acelerar o trabalho de parto, a menos que o atraso tenha sido diagnosticado. A OMS ${ }^{(10)}$ afirma ainda que, quando a intervenção precoce com ocitocina é utilizada antes da confirmação do atraso no trabalho de parto, pode haver um risco aumentado de hiper estimulação uterina, mudanças na frequência cardíaca do feto e resultados maternos e neonatais sombrios.

Quanto à episiotomia, constatou-se que a frequência de realização foi menor do que na pesquisa realizada pela Fundação Instituto Oswaldo Cruz (FIOCRUZ) ${ }^{(11)}$, que revelou essa prática rotineira em 56\% e em quase 75\% das primíparas de todo o país. A prática da episiotomia incorporou-se à rotina da assistência ao parto desde o início do século passado, com as seguintes intenções: reduzir o dano causado pela laceração natural do períneo; prevenir incontinência urinária e fecal; proteger o neonato do trauma do 
parto. Entretanto, nenhuma evidência constatou os riscos e os benefícios dessas condutas ${ }^{(3)}$.

A episiotomia é realizada rotineiramente pelos profissionais para acelerar o período expulsivo $^{(12)}$. Neste estudo, apesar de a taxa de realização de episiotomia estar abaixo do encontrado em outras pesquisas, ainda é considerada alta, pois, em alguns países onde se utilizam boas práticas na assistência ao parto, esse procedimento é realizado em menos de 10\% dos partos normais ${ }^{(13)}$.

Desse modo, a realização da amniotomia, a administração de ocitocina endovenosa e a episiotomia, intervenções comuns nas maternidades do Brasil para redução da duração do trabalho de parto e parto, precisam ser melhor avaliadas, de acordo com as evidências científicas mais recentes $^{(14)}$.

No que diz respeito ao período de internação em sala de parto, a admissão precoce na fase latente do trabalho de parto (dilatação $<4 \mathrm{~cm}$ ), deve ser evitada, por constituir um fator predisponente para a realização de intervenções. Entende-se que, quanto mais tarde for realizada a admissão, menor a possibilidade de haver intervenções, devido ao período em que a parturiente encontra-se no ambiente hospitalar ${ }^{(15)}$.

$\mathrm{Na}$ pesquisa realizada, ficou evidente que as parturientes em trabalho de parto ativo no período menor ou igual a 5 horas passaram por mais intervenções do que as parturientes com mais de 5 horas na fase ativa. A captação dessas informações dos prontuários de parturientes classificadas como gestantes de risco habitual, possibilitou a compreensão de que essas intervenções foram desnecessárias.

Nesse aspecto, destaca-se a importância de as instituições pautarem seus protocolos nas Diretrizes Nacionais de Assistência ao Parto Normal do Ministério da Saúde, e os profissionais adotarem o modelo obstétrico humanizado, que orienta a redução das intervenções sem indicação.

Ressalta-se ainda a necessidade de os profissionais fornecerem orientações às parturientes desde o pré-natal até a hospitalização, sobre indicações, riscos e benefícios das intervenções durante o trabalho de parto e parto, para que essas manifestem sua decisão de concordância ou negativa e autorizem ou não a realização dos procedimentos.

Essas considerações permitiram o entendimento de que as limitações deste estudo consistem na escassez de evidências científicas recentes com esse enfoque, para que se pudesse ampliar as discussões e apresentar comparações e proposições mais abrangentes.

\section{Conclusão}

A realização deste estudo possibilitou descrever a relação entre as intervenções realizadas durante o trabalho de parto e parto com a duração da fase ativa. As intervenções obstétricas avaliadas foram a amniotomia, a episiotomia e o uso de ocitocina endovenosa. Assim, parturientes na fase ativa de trabalho de parto com menos de 5 horas tiveram mais intervenções do que parturientes com mais de 5 horas.

Constatou-se que as intervenções realizadas não ocorreram na fase ativa do trabalho de parto prolongado ou por indicação que caracterizasse um parto de alto risco, à medida que, nos critérios de inclusão, considerou-se a seleção de prontuários de puérperas que tiveram parto de risco habitual.

Nesse sentido, entendeu-se que as intervenções, de acordo com a amostra selecionada, não se justificaram, pois, consoante as evidências científicas, quanto menor a duração da fase ativa do trabalho de parto, menor a proporção de parturientes passarem por intervenções.

Essas considerações possibilitam recomendar-se que os profissionais orientem as gestantes e seus acompanhantes sobre os riscos a curto e longo prazo das intervenções realizadas sem a devida indicação. Assim, espera-se que as parturientes sejam protagonistas do parto e tenham o poder de decisão informada, além de colaborarem no processo de redução das intervenções em prol do cuidado obstétrico humanizado.

Recomenda-se a implantação efetiva de protocolos assistenciais com base nas Diretrizes Nacionais de Assistência ao Parto e Nascimento, do Ministério da Saúde, além de educação 
permanente para a equipe multiprofissional, visando a adequação das práticas obstétricas ao modelo obstétrico humanizado.

\section{Colaborações:}

1 - concepção, projeto, análise e interpretação dos dados: Elisângela da Silva Ferreira, Luana Rocha Pereira e Ingrid Nicolle Monteiro Barros;

2 - redação do artigo e revisão crítica relevante do conteúdo intelectual: Elisângela da Silva Ferreira, Luana Rocha Pereira, Ingrid Nicolle Monteiro Barros, Márcia Simão Carneiro e Lorena Saavedra Siqueira;

3 - aprovação final da versão a ser publicada: Gilcynara Maria Moura Rodrigues, Elisângela da Silva Ferreira, Luana Rocha Pereira, Ingrid Nicolle Monteiro Barros, Márcia Simão Carneiro e Lorena Saavedra Siqueira.

\section{Referências}

1. Santos AHL, Nicácio MC, Pereira ALF, Oliveira TCM, Progianti JM. Práticas de assistência ao parto normal: formação na modalidade de residência. Rev enferm UFPE on line. 2017 jan;11(1):1-9. DOI: 10.5205/reuol.9963-88710-2-CE1101201701

2. Brasil. Ministério da Saúde. Secretaria de Ciência, Tecnologia e Insumos Estratégicos. Departamento de Gestão e Incorporação de Tecnologias em Saúde. Diretrizes nacionais de assistência ao parto normal: versão resumida [Internet]. Brasília (DF); 2017 [cited 2018 Jan 30]. Available from: http:// bvsms.saude.gov.br/bvs/publicacoes/diretrizes_ nacionais_assistencia_parto_normal.pdf

3. Leal MC, Pereira APE, Domingues RMSM, Theme Filha MM, Dias MAB, Pereira MN, et al. Intervenções obstétricas durante o trabalho de parto e parto em mulheres brasileiras de risco habitual. Cad Saúde Pública. 2014;30(suppl.1):S17-S32. DOI: 10.1590/0102-311X00151513

4. Nucci M, Nakano AR, Teixeira LA. Ocitocina sintética e a aceleração do parto: reflexões sobre a síntese e o início do uso da ocitocina em obstetrícia no Brasil. Hist cienc saúde. 2018 out.-dez;25(4):979-98.

5. World Health Organization. Intrapartum care for a positive childbirth experience [Internet].
Geneva; 2018 [cited 2019 Jan 15]. Available from: https://apps.who.int/iris/bitstream/handle/ $10665 / 260178 / 9789241550215$ - eng . pdf; jsessionid=40A5A 0D63533323E 1613 FD65828DD523?sequence $=1$

6. American College of Obstetricians and Gynecologists. ACOG Practice Bulletin $\mathrm{N}^{\circ}$. 198: Prevention and Management of Obstetric Lacerations at Vaginal Delivery. Obstet Gynecol. 2018 Sep;132(3):e87-e102. DOI: 10.1097/ AOG.0000000000002841

7. Corrêa Junior MD, Passini Júnior R. Selective Episiotomy: Indications, Techinique, and Association with Severe Perineal Lacerations. Rev Bras Ginecol Obstet. 2016 Jun;38(6):301-7. DOI: 10.1055/s-0036-1584942

8. Vogt SE, Silva KS, Dias MAB. Comparação de modelos de assistência ao parto em hospitais públicos. Rev Saúde Pública [online]. 2014;48(2):30413. DOI: $10.1590 / S 0034-8910.2014048004633$

9. Hidalgo-Lopezosa P, Hidalgo-Maestre M, Rodríguez-Borrego MA. Labor stimulation with oxytocin: effects on obstetrical and neonatal outcomes. Rev Latino-Am Enfermagem. 2016;24:e2744. DOI: 10.1590/1518-8345.0765.2744

10. Organização Mundial de Saúde. Recomendações para o aumento do trabalho de parto [Internet]. Genebra; 2015 [cited 2018 Jan 30]. Available from: http://www.who.int/reproductivehealth

11. Fundação Instituto Oswaldo Cruz. Nascer no Brasil. Inquérito nacional sobre parto e nascimento [Internet]. Rio de Janeiro; 2014 [cited 2018 Jan 8]. Available from: http://www.ensp.fiocruz. br/portal-nsp/informe/site/arquivos/anexos/ nascerweb.pdf

12. Carniel F, Vital DS, Souza TDP. Episiotomia de rotina: necessidade versus violência obstétrica. J nurs health. 2019;9(2):e199204. DOI: 10.15210/ JONAH.V9I2.14425

13. Violência Obstétrica "Parirás com dor". Dossiê elaborado pela Rede Parto do Princípio para a CPMI da Violência Contra as Mulheres [Internet]. Brasília (DF); 2012 [cited 2019 Jan 15]. Available from: https://www.senado.gov.br/comissoes/ documentos/SSCEPI/DOC\%20VCM\%20367.pdf

14. Brasil. Ministério da Saúde. Secretaria de Ciência, Tecnologia e Insumos Estratégicos. Diretriz Nacional de Assistência ao Parto Normal. Relatório de Recomendação. Brasília (DF); 2016. 
Luana Rocha Pereira, Gilcynara Maria Moura Rodrigues, Elisângela da Silva Ferreira, Ingrid Nicolle Monteiro Barros, Marcia Simão Carneiro, Lorena Saavedra Siqueira

15. Reis TR, Zamberlan C, Quadros JS, Grasel JT, Moro ASS. Enfermagem obstétrica: contribuições às metas dos Objetivos de Desenvolvimento do Milênio. Rev Gaúcha Enferm [online]. 2015; 36(n.spe):94-101. DOI: 10.1590/1983-1447.2015. esp. 57393
Recebido: 3 de setembro de 2019

Aprovado: 21 de outubro de 2019

Publicado: 29 de janeiro de 2020

A Revista Baiana de Enfermagem utiliza a Licença Creative Commons - Atribuição-NãoComercial 4.0 Internacional. https://creativecommons.org/licenses/by-nc/4.0/ Este artigo é de acesso aberto distribuído sob os termos da Licença Creative Commons (CC BY-NC). Esta licença permite que outros remixem, adaptem e criem a partir do seu trabalho para fins não comerciais. Embora os novos trabalhos tenham de lhe atribuir o devido crédito e não possam ser usados para fins comerciais, os usuários não têm de licenciar esses trabalhos derivados sob os mesmos termos. 